\title{
Graph Neural Networks to Advance Anticancer Drug Design
}

\author{
Asmaa Rassil $^{1(\bowtie)}$, Hiba Chougrad ${ }^{2}$, and Hamid Zouaki ${ }^{1}$ \\ 1 Faculty of Science, Laboratory of Computer Science and Mathematics \\ and their Applications, University Chouaib Doukkali, El Jadida, Morocco \\ asmaarassil@gmail.com, hamid_zouaki@yahoo.fr \\ 2 Laboratory of Intelligent Systems, Georesources and Renewable Energies, \\ National School of Applied Sciences, University Sidi Mohamed Ben Abdellah, \\ $\mathrm{Fez}, \mathrm{Morocco}$ \\ chougrad.hiba@gmail.com
}

\begin{abstract}
Predicting the activity of chemical compounds against cancer is a crucial task. Active chemical compounds against cancer help pharmaceutical drugs producers in the conception of anticancer medicines. Still the innate way of representing chemical compounds is by graphs, the machine learning algorithms can not handle directly the anticancer activity prediction problems. Dealing with data defined on a non-Euclidean domain gave rise to a new field of research on graphs. There has been many proposals over the years, that tried to tackle the problem of representation learning on graphs. In this work, we investigate the representation power of Node2vec for embedding learning over graphs, by comparing it to the theoretical framework Graph Isomorphism Network (GIN). We prove that GIN is a deep generalization of Node2vec. We then exert the two models Node2vec and GIN to extract regular representations from chemical compounds and make predictions about their activity against lung and ovarian cancer.
\end{abstract}

Keywords: Graph neural networks - Transductive learning $\cdot$ Inductive learning $\cdot$ Representation vectors $\cdot$ Anticancer activity prediction

\section{Introduction}

Application domains of Artificial Intelligence (AI) became concerned in the last few years with treating complex problems defined on irregular data structure as graphs. The conception of pharmaceutical drugs $[9,18,29]$ through the prediction of chemical compounds activities against cancer $[22,23]$ need more than tabular representations to be solved. The natural way of modeling molecular data for an anticancer activity prediction problem is by graphs. Representing chemical compounds in the shape of graphs keep the structure of the data unchanged, and so no information from the initial collected data will get lost. Graphs are 
irregular types of data which are defined on non-Euclidean domains, therefore, all the classic machine learning algorithms cannot be directly applied on this type of structure [4]. To deal with such complex data, a number of works on representation learning on graphs have been proposed and proved to be effective in resolving these tasks. These representation approaches aim to map nodes or the entirely given graph to an embedding space of low-dimension (Fig. 1). The embedding vectors are supposed to summarize the information on top of nodes and their surrounding neighbors [13]. To do representation learning on graphs two types of methods are proposed transductive and inductive learning. Inductive approaches $[2,7,11,13,28]$ for representation learning on graphs do not learn the embedding vectors directly from the graph but use instead the training graphs to learn a parametric encoding function to generate embeddings for unseen graphs. Transductive approaches $[1,3,6,19,21,26]$ associate input graphs to embedding matrices of $\mathrm{N}$ rows and $\mathrm{d}$ columns. The parameter $\mathbf{N}$ refers to the number of nodes in the given graph and $\mathbf{d}$ refers to the dimensionality of the embedding space. Therefore, by transforming the irregular structure of graphs into Nxd matrices, we can straightforwardly apply Machine Learning algorithms to the data. In this work, we investigate the representation power of Node2vec for embedding learning over graphs, by comparing it to the inductive theoretical framework Graph Isomorphism Network (GIN) that proved to be equivalent to the Weisfeiler-Lehman (WL) test of isomorphism. We demonstrate theoretically that GIN is a deep generalization of Node2vec. We employ Node2vec and GIN models to define regular representations of chemical compounds represented by graphs and use these regular representations to predict the activities of the corresponding chemical compounds against lung and ovarian cancer in NCI1 and NCI109 $[22,23]$ datasets respectively.

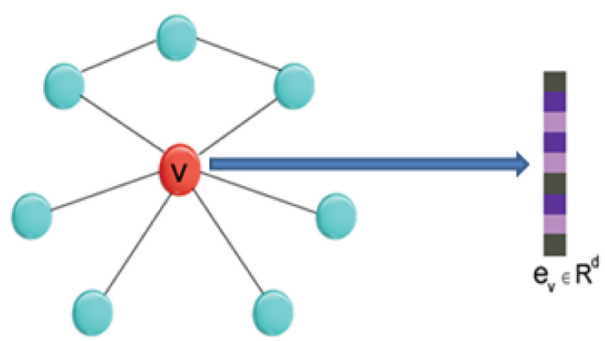

Fig. 1. Embedding learning. Each node $v$ in the graph is mapped to a d-dimensional embedding vector $e_{v}$ which summarizes the local information of the nodes around $v$.

\section{Problem Formulation}

A graph $\mathbf{G}=(\mathbf{V}, \mathbf{E})$ is defined by the vertex set $\mathbf{V}=\left\{v_{i}: i=1,2, \ldots, N\right\}$ and the edges $e_{i j} \in \mathbf{E}$ connecting pairs of nodes $v_{i}$ and $v_{j}$ in the graph. For each 
node $v$ in the graph, we note its neighbors set by $\mathcal{N}(v)=\{u:(u, v) \in E\}$. The adjacency matrix $\mathbf{A}=\left(a_{i j}\right)_{1 \leq i \leq N, 1 \leq j \leq N}$ outlines the graph structure by setting $a_{i j}=1$ for connected nodes $e_{i j} \in E$ and $a_{i j}=0$ if $e_{i j} \notin E$.

\subsection{Graph Embedding}

Graph embedding frameworks $[1,3,6,19,21,26]$ are unsupervised approaches for learning on graph structured data. The main purpose of network embedding is to map input graphs to a regular low dimensional space where the use of standard machine learning algorithms become feasible $[5,8,14]$. To preserve the graph topology and the structural information on graphs in the embedding space, the network embedding variants propose pairwise similarity measures upon the graph structure $S_{G}(u, v)$ and learn the node embeddings by preserving the defined similarity measure in the embedding space $S_{E}\left(e_{u}, e_{v}\right)$ through the following loss function:

$$
L\left[S_{G}(u, v), S_{E}\left(e_{u}, e_{v}\right)\right]
$$

where $e_{v} \in \mathbf{R}^{d}$ is the corresponding embedding vector of the node $v \in \mathbf{V}$ in the embedding space $E=\mathbf{R}^{d}$ and $L($.$) is a loss function.$

Two principal branches of graph embedding are proposed in the literature [14] matrix factorization $[1,3,6,19]$ and random walk [12,20]. Matrix factorization approaches define the proximity measure over the graph $S_{G}(u, v)$ either by an adjacency-based pairwise proximity measure $\left(S_{G}(u, v)=A_{u v}\right)$ [1], or by multi-hop pairwise proximity measure captured by the k-th power of the adjacency matrix of the graph $\left(S_{G}(u, v)=A_{u v}^{k}\right)[6]$ (see Fig. 2). The adjacency-based approaches push adjacent nodes $(u, v) \in E$ in the graph $S_{G}(u, v)=A_{u v}=1$ to be equal in the embedding space $S_{E}(u, v)=e_{v}^{T} e_{u}=1$ through the following loss function:

$$
\left\|A_{u v}-e_{v}^{T} e_{u}\right\|_{2}^{2}
$$

Multi-hop pairwise proximity measures $\left(S(u, v)=A_{u v}^{k}\right)[6]$ push adjacent nodes captured by $A^{k}$ to be equal in the embedding space $S_{E}(u, v)=e_{v}^{T} e_{u}$ through the following loss function:

$$
\left\|A_{u v}^{k}-e_{v}^{T} e_{u}\right\|_{2}^{2}
$$

Thus, nodes located in k-hop distance from each other, will have similar embedding vectors. Random walk $[12,20]$ approaches for graph embedding are heuristic methods for embedding learning. To learn embeddings, authors in [12, 20 ] optimize the probability of visiting nodes $v$ that tend to co-occur in random walks on the graph starting from a node $u$

$$
p(v \mid u)=\frac{\exp \left(e_{v}^{T} e_{u}\right)}{\sum_{v_{i} \in \mathbf{V}} \exp \left(e_{v}^{T} e_{v_{i}}\right)}
$$

Matrix factorization gives a deterministic definition of similarity upon the graph. Otherwise, random walk methods adopt a stochastic proximity measure 


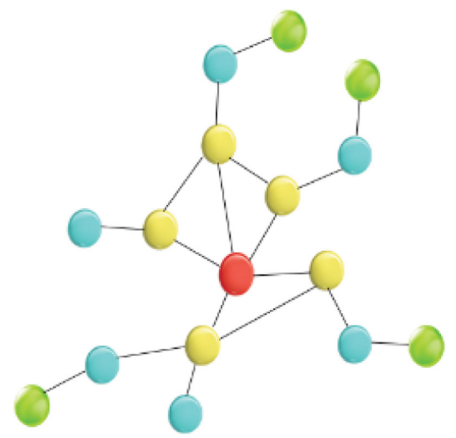

source node

1-hop neighbors

Adjacency based similarity

2-hop neighbors

Multi-hop based similarity

3-hop neighbors

Multi-hop based similarity

Fig. 2. For every node (i.e. source node), matrix factorization approaches learn embedding vectors of source nodes based on information from local neighbors (i.e. 1-hope neighbors) or distant neighbors.

based on randomly generated walks on the graph. Through two searching parameters $p$ and $q$, Node2vec [12] controls the propagation of the generated walk. For $p>\max (q, 1)$ we reduce the probability of sampling an already visited node in the next two steps, therefore distant nodes from a source node $u$ are pushed to have similar embeddings [12]. However, for $p<\min (q, 1)$, the walk stays local, then closest nodes to the source are pushed to have similar embeddings (Fig. 3).

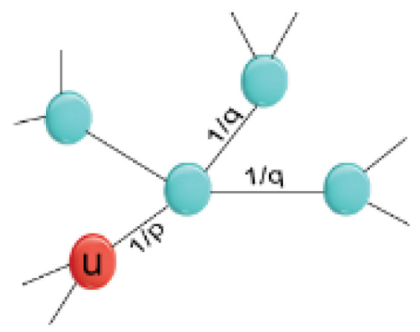

Fig. 3. The parameters $p$ and $q$ control the propagation of the generated walk on the graph. For small values of $p$, local neighbors are likely to be visited. However, when setting the parameter $q$ to a small value, distant nodes tend to co-occur in the random walk

\subsection{Graph Neural Networks}

Contrary to graph embedding, graph neural networks (GNNs) $[2,7,11,13,28]$ are deep and inductive approaches for representation learning on graphs. Through an end-to-end network, GNNs learn jointly the embeddings or representation vectors of the nodes and solve the defined problem on the graph structure. Thus, the representation vectors are learned based on the intrinsic topology of the graph and the task we aim to solve. The representation learning process involves 
two steps (i) aggregating neighboring nodes then (ii) combining them with the node being processed through the following recursive schema:

$$
\begin{aligned}
& a_{v}^{(k)}=A G G R E G A T E^{(k)}\left(\left\{h_{u}^{(k-1)} \quad: u \in \mathcal{N}(v)\right\}\right) \\
& h_{v}^{(k)}=C O M B I N E^{(k)}\left(h_{v}^{(k-1)}, a_{v}^{(k)}\right)
\end{aligned}
$$

where $h_{v}^{(k)}$ is the learned representation vector of node $v$ in the kth layer of the network. Variants of aggregation and combination functions give the GNN variants. Graph Isomorphism Network (GIN) [25] is a theoretical variant of GNNs which combines the aggregation and combination step in the following formula:

$$
h_{v}^{(k)}=M L P^{(k)}\left[\left(1+\epsilon^{(k)}\right) h_{v}^{(k-1)}+\sum_{u \in \mathcal{N}(v)} h_{u}^{(k-1)}\right]
$$

where $\epsilon^{(k)}$ is a model parameter. Graph Isomorphism Network (GIN) [25] is as powerful as the Weisfeiler-Lehman (WL) test of isomorphism [24]. Thus, the learned graph representation vectors are as powerful as WL in capturing differences of graph topologies.

\section{Deep Generalization of Node2vec}

Learning embedding vectors by maximizing the likelihood of visiting the neighbors generated through a combination of Breadth-first Sampling (BFS) and Depth-first Sampling (DFS) strategies bestow Node2vec [12] with an impressive representation power.

When setting the parameter $p$ to a small value (Fig. 3), nodes belonging to similar communities [27] in a graph are forced to learn approximately the same embeddings. However, when setting the parameter $q$ to a small value (Fig. 3), nodes representing the same structural role in different communities [15] are pushed to learn similar embeddings. Therefore, according to the given task Node2vec [12] is able to learn suitable embeddings.

In this work, we demonstrate the analogy between Node2vec [12] and the powerful theoretical framework GIN [25]. And, we prove that Node2vec is closely equivalent to the Weisfeiler-Lehman(WL) test of isomorphism [24].

We consider a shallow neural network with one hidden layer $\mathbf{h}$ as described in Fig. 4. Given a node $u$, we maximize the probability of visiting a node $v \in \mathcal{N}(v)$ by generating a random walk starting from $u$. We initialize the embedding of $u$ by its corresponding column $a_{u} \in \mathbf{R}^{N}$ in the adjacency matrix $\hat{A}=A+\mathbb{I}_{N}$, then we feed it as input to the network (Fig. 4). We denote the $N \times d$ matrices corresponding to the model parameters in the input-to-hidden and the hiddento-output sections of the network by $W$ and $W^{\prime}$ respectively. We assume that $W$ and $W^{\prime}$ are embedding matrices of the input graph. Each row of $W$ and $W^{\prime}$ is a d-dimensional embedding vector $e_{v}$ of a node $v \in \mathbf{V}$.

In the forward pass, 


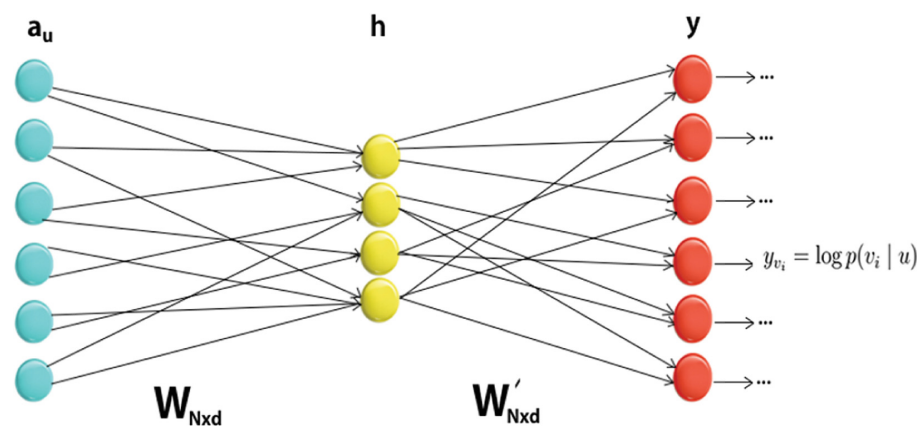

Fig. 4. Unsupervised representation Learning on graphs. Starting from a source node $u$ defined by its corresponding column in the adjacency matrix $\hat{A}=A+\mathbb{I}_{N}$, we update the embedding of $u$ by maximizing the probability of visiting the neighbors $v \in \mathcal{N}(u)$ generated by a random walk. The embedding we are looking for are defined by the parameters $W$ and $W^{\prime}$ of the model.

$$
W^{T} a_{u}=\sum_{v \in \mathcal{N}(u) \cup u} W_{., k}^{T}=e_{u}+\sum_{v \in \mathcal{N}(u)} e_{v}^{T}=h_{u}=\mathbf{h}
$$

the updated embedding vector $h_{u} \in \mathbf{R}^{d}$ of the input node $u$ is a summation of the embedding vectors $e_{u}$ of $u$ and its surrounding neighbors $\left\{e_{v}: v \in \mathcal{N}(u)\right\}$ given by the embedding matrix $W$. Node2vec adopts a sum-aggregator to aggregate neighboring nodes and utilizes the collected information to update the initial node embedding $a_{u}$. Thus, we may proclaim that node2vec is a shallow approximation of the deep and inductive Graph Isomorphism Network GIN (Eq. 6).

The output of the network before applying activation is,

$$
z_{i}=W_{., i}^{\prime T} \mathbf{h}=W_{., i}^{\prime} h_{u}=h_{v_{i}}^{T} h_{u} \quad \forall i=1, \ldots, N
$$

using a log-softmax activation function $\Phi($.$) , we obtain (Eq. 3) corresponding to$ Node2vec:

$$
\Phi\left(h_{v_{i}}^{T} h_{u}\right)=\log p\left(v_{i} \mid u\right)=\log \frac{\exp \left(h_{v_{i}}^{T} h_{u}\right)}{\sum_{v \in \mathbf{V}} \exp \left(h_{v}^{T} h_{v}\right)}
$$

We can conclude that, Node2vec learns embedding vectors of the graph nodes using approximately the same process of one layer GIN. Now, considering that GIN can capture and distinguish between different graph topologies as would the WL test of isomorphism, we may assert that embeddings learned by Node2vec will also be powerful in capturing differences of graph topologies.

\section{Experiments and Results}

To predict anticancer activity of chemical compounds against lung and ovarian cancer, we investigate the representation power of Node2vec for embedding learning on graphs and two variants of the inductive Graph Isomorphism Network 
(GIN) using two benchmark datasets NCI-1 and NCI-109 for lung and ovarian cancer respectively. In the following paragraphs we provide a description of the datasets, details about the models architecture and configurations, and the obtained results achieved by each model for predicting anticancer activity.

\subsection{Datasets}

The NCI-1 and NCI-109 graph datasets [22,23] are provided by the National Cancer Institute. Each NCI dataset contains thousands of chemical compounds modelled as graphs. The atoms are represented as nodes and bonds as edges in the graph representation of the chemical compounds (Fig. 5). Statistics about NCI-1 and NCI-109 graph datasets are reported in Table 1.

NCI-1 dataset contains two categories of chemical compounds active and nonactive against the non-small cell lung cancer. Active compounds are labeled by +1 and non-active by -1 . NCI-109 dataset contains two categories of chemical compounds active and non-active against ovarian cancer. Active compounds are labeled by +1 and non-active by -1 .

Table 1. Datasets statistics. The table summarizes the number of graphs, the average number of nodes, the average number of edges and the number of node labels in NCI-1 and NCI-109 datasets.

\begin{tabular}{l|l|l|l|l}
\hline \multirow{2}{*}{ Datasets } & \multicolumn{4}{|l}{ Dataset statistics } \\
\cline { 2 - 5 } & \# Graphs & \# Nodes(avg.) & \# Edges(avg.) & \# Node Labels \\
\hline NCI-1 & 4110 & 29.8 & 32.3 & 37 \\
\hline NCI-109 & 4127 & 29.6 & 32.1 & 38 \\
\hline
\end{tabular}

\subsection{Implementation Details and Results}

To predict the anticancer activity of chemical compounds against lung and ovarian cancer, we train the embedding model Node2vec [12] on NCI-1 and NCI109 datasets with the following hyper-parameters: embedding dimensionality $\in\{7,12\}$, random walk length $\in\{3,4\}$, size of neighbors set (i.e the context size $) \in\{5,7\}$ and for each node we generate 10 random walks.

To train the deep Graph Isomorphism Network (GIN) [25] on NCI-1 and NCI109 datasets, we use two variants of the model using (1) multilayer perceptron and (2) single-layer perceptron as combination functions of neighboring node representations. To build the anticancer activity prediction model, we use five GIN layers each followed by batch normalization [16]. We learn the model parameters by optimizing the cross-entropy loss between the predicted labels and the true labels using the Adam optimizer [17]. We tune the following hyper-parameters: batch size $\in\{32,42\}$, hidden units $\in\{16,32\}$, learning rate $\in\{0.01,0.001\}$ and dropout coefficient $\in\{0,0.2\}$. All implementations were carried out using PyTorch Geometric [10] and the Cuda enabled GPU 12GB NVIDIA Tesla K80. 
<smiles>Nc1ccccc1</smiles><smiles>N#CC1C=CCCCC1</smiles>

Fig. 5. Graph representation of a chemical compound. The left figure represents a chemical compound and the right figure represents its corresponding graph representation where $\mathrm{C}$ refers to carbon atom, $\mathrm{H}$ to Hydrogen atom and $\mathrm{N}$ to Nitrogen atom.
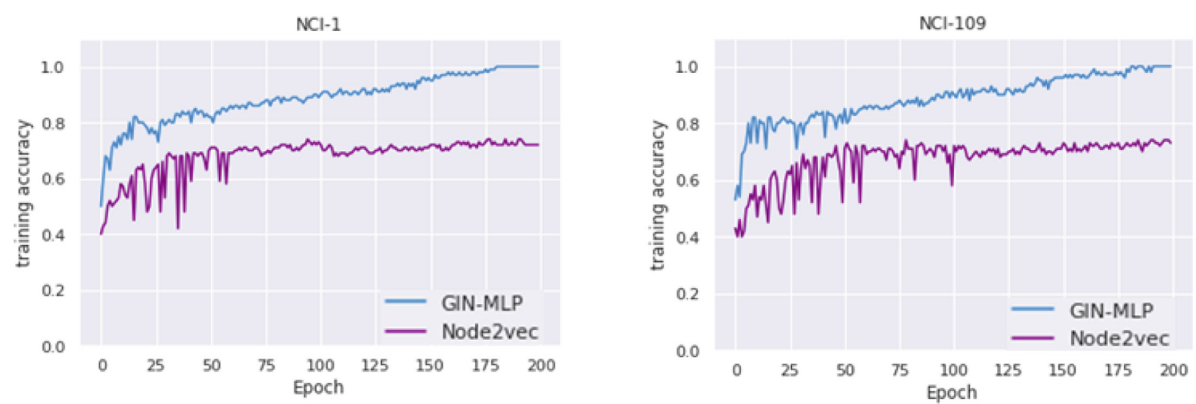

Fig. 6. Training set performance of GIN-MLP compared to Node2vec on NCI-1 and NCI-109 datasets over 100 epochs

Table 2. Binary-classification results (\%). The table reports the average and standard deviations of accuracies on binary-graph classification

\begin{tabular}{l|l|l|l}
\hline \multirow{2}{*}{ Datasets } & \multicolumn{3}{|l}{ Representation learning models } \\
\cline { 2 - 4 } & Node2vec [12] & GIN-1-Layer [25] & GIN-MLP [25] \\
\hline NCI-1 & $54.9 \pm 1.2$ & $83.0 \pm 3.5$ & $83.4 \pm 2.5$ \\
\hline NCI-109 & $51.4 \pm 2.3$ & $86.8 \pm 6.0$ & $85.0 \pm 6.7$ \\
\hline
\end{tabular}

Results reported in Table 2 and Fig. 6 show that GIN models perfectly fit the data and report approximately the same results for predicting the anticancer activity of chemical compounds on both NCI-1 (GIN-1-layer gives an average accuracy of $83.0 \pm 3.5 \%$ and GIN-MLP gives $83.4 \pm 2.5 \%$ ) and NCI-109 (GIN1-layer gives an average accuracy of $86.8 \pm 6.0 \%$ and GIN-MLP gives $85.0 \pm$ $6.7 \%$ accuracy) datasets, while Node2vec has a low and unstable performance on the two datasets (average accuracy of $54.9 \pm 1.2 \%$ on NCI-1 for lung cancer and $51.4 \pm 2.3 \%$ on NCI-109 for ovarian cancer). Those results are maybe due to the fact that GIN models are deep while Node2vec is a shallow model. 
Subsequently, we validate the representation power of Node2vec by comparing it theoretically to the Graph Isomorphism Network (GIN) [25] which is proved to be as powerful as the Weisfeiler-Lehman(WL) test of isomorphism [24] in capturing differences on graph structures and topologies. We use Node2vec and two variants of the GIN model to auspiciously predict the activity of chemical compounds against lung and ovarian cancer over NCI-1 and NCI-109 respectively. Chemical compounds predicted to be active against lung and ovarian cancer can then be decisive in the conception of pharmaceutical anticancer drugs.

\section{Conclusion}

In this work, we investigate the representation learning power of Graph Neural Networks on graph data structure. We explore two powerful models that operate on graphs (1) Node2vec and (2) Graph Isomorphism Network and derive that the latter is a deep and inductive extension of Node2vec. Thus, based on the aforementioned results we are able to apprehend the representation power of Node2vec.

Predicting effectively the activity of chemical compounds against cancer helps pharmaceutical drugs producers in the fabrication of anticancer medicines. We exploit Node2vec and GIN representation power over graph data to predict the activity of chemical compounds against lung and ovarian cancer and achieve remarkable results using GIN variants.

As a future work, we would like to define a new specially-designed deep learning framework for representation learning on chemical compounds modelled as graphs. We also plan to go beyond neighbor aggregation and adopt attention mechanisms to learn the connective strength between nodes.

\section{References}

1. Ahmed, A., Shervashidze, N., Narayanamurthy, S., Josifovski, V., Smola, A.J.: Distributed large-scale natural graph factorization. In: Proceedings of the 22nd International Conference on World Wide Web, pp. 37-48 (2013)

2. Atwood, J., Towsley, D.: Diffusion-convolutional neural networks. In: Advances in Neural Information Processing Systems, pp. 1993-2001 (2016)

3. Belkin, M., Niyogi, P.: Laplacian eigenmaps and spectral techniques for embedding and clustering. In: Advances in Neural Information Processing Systems, pp. 585591 (2002)

4. Bronstein, M.M., Bruna, J., LeCun, Y., Szlam, A., Vandergheynst, P.: Geometric deep learning: going beyond Euclidean data. IEEE Signal Process. Mag. 34(4), 18-42 (2017)

5. Cai, H., Zheng, V.W., Chang, K.C.C.: A comprehensive survey of graph embedding: problems, techniques, and applications. IEEE Trans. Knowl. Data Eng. 30(9), 1616-1637 (2018)

6. Cao, S., Lu, W., Xu, Q.: GraRep: learning graph representations with global structural information. In: Proceedings of the 24th ACM International on Conference on Information and Knowledge Management, pp. 891-900 (2015) 
7. Chen, J., Ma, T., Xiao, C.: FastGCN: fast learning with graph convolutional networks via importance sampling. arXiv preprint arXiv:1801.10247 (2018)

8. Cui, P., Wang, X., Pei, J., Zhu, W.: A survey on network embedding. IEEE Trans. Knowl. Data Eng. 31(5), 833-852 (2018)

9. De Cao, N., Kipf, T.: MolGAN: an implicit generative model for small molecular graphs. arXiv preprint arXiv:1805.11973 (2018)

10. Fey, M., Lenssen, J.E.: Fast graph representation learning with PyTorch geometric. In: ICLR Workshop on Representation Learning on Graphs and Manifolds (2019)

11. Gilmer, J., Schoenholz, S.S., Riley, P.F., Vinyals, O., Dahl, G.E.: Neural message passing for quantum chemistry. In: Proceedings of the 34th International Conference on Machine Learning, vol. 70, pp. 1263-1272. JMLR. org (2017)

12. Grover, A., Leskovec, J.: node2vec: scalable feature learning for networks. In: Proceedings of the 22nd ACM SIGKDD International Conference on Knowledge Discovery and Data Mining, pp. 855-864 (2016)

13. Hamilton, W., Ying, Z., Leskovec, J.: Inductive representation learning on large graphs. In: Advances in Neural Information Processing Systems, pp. 1024-1034 (2017)

14. Hamilton, W.L., Ying, R., Leskovec, J.: Representation learning on graphs: methods and applications. arXiv preprint arXiv:1709.05584 (2017)

15. Henderson, K., et al.: RolX: structural role extraction \& mining in large graphs. In: Proceedings of the 18th ACM SIGKDD International Conference on Knowledge Discovery and Data Mining, pp. 1231-1239 (2012)

16. Ioffe, S., Szegedy, C.: Batch normalization: accelerating deep network training by reducing internal covariate shift. arXiv preprint arXiv:1502.03167 (2015)

17. Kingma, D.P., Ba, J.: Adam: a method for stochastic optimization. arXiv preprint arXiv:1412.6980 (2014)

18. Li, Y., Vinyals, O., Dyer, C., Pascanu, R., Battaglia, P.: Learning deep generative models of graphs. arXiv preprint arXiv:1803.03324 (2018)

19. Ou, M., Cui, P., Pei, J., Zhang, Z., Zhu, W.: Asymmetric transitivity preserving graph embedding. In: Proceedings of the 22nd ACM SIGKDD International Conference on Knowledge Discovery and Data Mining, pp. 1105-1114 (2016)

20. Perozzi, B., Al-Rfou, R., Skiena, S.: DeepWalk: online learning of social representations. In: Proceedings of the 20th ACM SIGKDD International Conference on Knowledge Discovery and Data Mining, pp. 701-710 (2014)

21. Shen, X., Pan, S., Liu, W., Ong, Y.S., Sun, Q.S.: Discrete network embedding. In: Proceedings of the 27th International Joint Conference on Artificial Intelligence, pp. 3549-3555 (2018)

22. Shervashidze, N., Schweitzer, P., Leeuwen, E.J.V., Mehlhorn, K., Borgwardt, K.M.: Weisfeiler-Lehman graph kernels. J. Mach. Learn. Res. 12, 2539-2561 (2011)

23. Wale, N., Watson, I.A., Karypis, G.: Comparison of descriptor spaces for chemical compound retrieval and classification. Knowl. Inf. Syst. 14(3), 347-375 (2008)

24. Weisfeiler, B., Leman, A.: A reduction of a graph to a canonical form and an algebra arising during this reduction. Nauchno-Technicheskaya Informatsia 9, 12-16 (1968)

25. Xu, K., Hu, W., Leskovec, J., Jegelka, S.: How powerful are graph neural networks? In: International Conference on Learning Representations (ICLR) (2019)

26. Yang, H., Pan, S., Zhang, P., Chen, L., Lian, D., Zhang, C.: Binarized attributed network embedding. In: 2018 IEEE International Conference on Data Mining (ICDM), pp. 1476-1481. IEEE (2018) 
27. Yang, J., Leskovec, J.: Overlapping communities explain core-periphery organization of networks. Proc. IEEE 102(12), 1892-1902 (2014)

28. Ying, Z., You, J., Morris, C., Ren, X., Hamilton, W., Leskovec, J.: Hierarchical graph representation learning with differentiable pooling. In: Advances in Neural Information Processing Systems, pp. 4800-4810 (2018)

29. You, J., Liu, B., Ying, Z., Pande, V., Leskovec, J.: Graph convolutional policy network for goal-directed molecular graph generation. In: Advances in Neural Information Processing Systems, pp. 6410-6421 (2018) 